\title{
On the optical-infra-red continuum emission from equatorial discs of supergiant $B[e]$ stars
}

\begin{abstract}
John M. Porter
Astrophysics Research Institute, Liverpool John Moores University, Twelve Quays House, Egerton Wharf, Birkenhead, CH41 1LD, UK

Received 22 October 2001 / Accepted 19 November 2002

Abstract. Two models of the circumstellar disc around supergiant $\mathrm{B}[\mathrm{e}]$ stars are discussed: an equatorial wind model produced by wind bi-stability, and a Keplerian viscous disc model. Both models are successful in providing a site for dust formation once they have cooled sufficiently. However, the optical-infra-red continuum is calculated and it is found that both models have significant trouble in accounting for observations. In particular the optical-near-IR emission is accounted for, but the dust emission is underestimated by at least an order of magnitude. Variations in the structure of the models (the temperature variation with radius, the density structure and the dust opacity) are investigated to assess how (in)appropriate the standard models are for supergiant $\mathrm{B}[\mathrm{e}]$ star discs. Changing the temperature structure, and making simple dust opacity changes within the disc has little effect on the resultant continuum emission. By altering the density structure of the discs, the continuum may be accounted for by both models: the equatorial wind model requires a very flat density profile which is impossible to explain with any accelerating wind, and the viscous disc model's density structure is required to fall off less steeply with radius than would have been expected, although this may be explained from consideration of viscous processes in the disc. It is recognised that both theoretical interpretations have difficulties and unsolved problems.
\end{abstract}

Key words. stars: emission-line, Be - stars: supergiants - stars: circumstellar matter - stars: individual: R126 (HD 37974 )

\section{Introduction}

Observations of supergiant $\mathrm{B}[\mathrm{e}]$ stars (denoted $\mathrm{sgB}[\mathrm{e}]$ stars hereafter - see Lamers et al. 1998 for a classification system) show several features: infra-red (IR) continuum excesses and far-IR dust emission (e.g. see Zickgraf 1992); broad $\left(\sim 10^{3} \mathrm{~km} \mathrm{~s}^{-1}\right)$ UV resonance lines; in the optical there are strong Balmer lines ( $\mathrm{H} \alpha$ equivalent widths of $>10^{2} \AA$ ), and narrow $\left(10 \mathrm{~s}\right.$ of $\left.\mathrm{km} \mathrm{s}^{-1}\right)$ permitted and forbidden line emission. These stars have typical stellar parameters of $M_{*} \sim 30$ $70 M_{\odot}, R_{*} \sim 30-100 R_{\odot}, T_{\text {eff }} \sim 15000-30000 \mathrm{~K}$ (Zickgraf et al. 1986).

Zickgraf et al. (1985) proposed that the observations could be explained with a multicomponent wind structure: a fast linedriven wind (appropriate for a hot star) over the poles and a dense disc of outflowing gas in the equatorial plane. This geometry has received some corroboration from polarimetry (Zickgraf \& Schulte-Ladbeck 1989; Oudmaijer et al. 1998).

Whilst there is a good physical explanation of the fast polar wind, (Castor et al. 1975) the formation mechanism (and indeed the structure) of the dense equatorial flow is still largely unknown. An excellent attempt at explaining the equatorial flow has been made by Lamers \& Pauldrach (1991 - rotationally-induced wind bi-stability), which has been recently advanced by Pelupessy et al. (2000) who suggest that

\footnotetext{
^ e-mail: jmp@astro.livjm.ac.uk
}

wind compression due to its rotation (Bjorkman \& Cassinelli 1993) may also play a part in the generation of the disc (although wind compression was found by Owocki et al. 1996 and also by Petrenz \& Puls 2000 to be strongly inhibited in rotating winds). Zickgraf et al. (1996) fit a wind-like flow with a "beta" type velocity law to observations of the UV metallic lines of R50 and Hen S22 (interpreted as edge-on stars) and found acceptable fits with wind terminal velocities of $60-80 \mathrm{~km} \mathrm{~s}^{-1}$.

Oudmaijer et al. (1998) present a hydrodynamical model of the flow around HD 87643 in which a line-driven wind is driven partly by luminosity from a Keplerian reprocessing disc. As well as producing a fast polar wind, their model produces a region several scale-heights above the equatorial plane which has intermediate velocities $\left(\sim 100 \mathrm{~km} \mathrm{~s}^{-1}\right)$, and hence they point to this region being responsible for the permitted line emission. However, they did not discuss the disc formation, being more concerned with the intermediate and fast radiatively driven flows.

Given the current uncertainties in the sgB[e] stars' disc origin, a model for an equatorial wind produced by the bi-stability mechanism, and also a model for a viscous Keplerian disc (similar to that currently gaining acceptance in the classical-Be star community) are directly compared. The aim of this study is to assess if either model can be ruled out, or (at least) if one model can be strongly favoured over the other as the more likely disc structure of $\mathrm{sgB}[\mathrm{e}]$ stars. As a first step, the optical-IR spectral energy distribution is calculated and compared to observations 
(the line emission is returned to in the discussion). This is not a trivial task as a successful model must also provide a site for the formation of dust. In Sect. 2 the density and velocities of both the equatorial wind model and the viscous disc model are discussed, along with a heuristic argument for the temperature profile. The possibility of the flow forming dust is considered in Sect. 3. In Sect. 4 the continuum emission is calculated and its variation with relevant parameters presented. A discussion is given in Sect. 5 and conclusions in Sect. 6.

\section{The models}

The main concern of this study is to examine if either model can account for the continuum observations of $\mathrm{sgB}[\mathrm{e}]$ stars. Most previous work has examined aspects of wind models (for example Bjorkman 1998, presents results of a wind compression model) whilst this is the first time a viscous disc model has been applied to $\mathrm{sgB}[\mathrm{e}]$ stars. During the course of the study, values for the stellar parameters, fast polar wind etc. are taken from the Large Magellanic Cloud star R126: $M_{*} \approx 40 M_{\odot}$, $R_{*} \approx 70 R_{\odot}, T \approx 22500 \mathrm{~K}$ (Zickgraf et al. 1985), the fast polar wind parameters vary widely in the literature: a massloss rate of $\dot{M}_{\mathrm{w}}=10^{-6}-10^{-5} M_{\odot} \mathrm{yr}^{-1}$, and terminal velocity of $v_{\infty} \approx 1800 \mathrm{~km} \mathrm{~s}^{-1}$ was calculated by Zickgraf et al. (1985), whilst Bjorkman (1998) gives $\dot{M}_{\mathrm{w}}=4.6 \times 10^{-5} M_{\odot} \mathrm{yr}^{-1}$ and $v_{\infty}=650 \mathrm{~km} \mathrm{~s}^{-1}$ from Kudritzki et al.'s (1989) "cooking recipe". The adopted nomenclature used in this paper is that mass-loss rates will be quoted as a mass-flux multiplied by $4 \pi$ i.e. the rate is quoted as if the mass-loss was present over the whole star. This should be particularly noted when considering the mass flux through the disc.

\subsection{Density and velocity structure}

\subsubsection{Equatorial wind model}

The popular model for the equatorial density enhancement of $\mathrm{sgB}[\mathrm{e}]$ circumstellar media is one based on radiatively-driven winds. Support for this model came in 1991 when the bistability model was presented, in which the stellar wind parameters (mass-loss rate and velocity) "jump" over a band centered on the equator of the star as the driving species and lines change at temperatures in the region of $25000 \mathrm{~K}$ due to the changing conditions at the photosphere from a gravity-darkened fast rotating star (Lamers \& Pauldrach 1991; Vink et al. 1999, and Pelupessy et al. 2000) The model is characterised by a latitudinal varying mass flux rate $\dot{M}(\theta)$ and wind speed $v_{\mathrm{r}}(\theta)$. At the jump latitude $\theta, \dot{M}(\theta)$ increases and $v_{\mathrm{r}}$ decreases producing a large increase in density $\rho(\theta) \propto \dot{M}(\theta) / r^{2} v_{\mathrm{r}}$. Within this high density, low velocity region (representing the "disc"), the radial velocity is $v_{\mathrm{r}, \mathrm{d}}$ and density is $\rho_{\mathrm{d}}$. The model requires that the star is rotating at a substantial fraction $(>50 \%)$ of its critical speed (where centrifugal forces balance gravity). The azimuthal velocity of the wind in the disc $v_{\phi, \mathrm{d}}$ decreases with radius from the star due to angular momentum conservation and so is not very important dynamically in the disc. The disc thickness is determined by the value of $\theta$ where the bi-stability jump occurs, and so depends on the rotation rate and how close the effective temperature is to the jump temperature.

Within the disc, the density and radial and azimuthal velocities follow the form

$\rho_{\mathrm{d}}=\frac{\dot{M}_{\mathrm{d}}}{4 \pi r^{2} v_{\mathrm{r}, \mathrm{d}}}$
$v_{\mathrm{r}, \mathrm{d}}=w(r) v_{\infty, \mathrm{d}}$
$v_{\phi, \mathrm{d}}=v_{\phi 0}\left(\frac{r}{R_{*}}\right)^{-1}$

where $v_{\phi 0}$ is the rotational speed at the stellar surface and again note the disc mass-loss rate is quoted as if this mass loss applied over the whole star - the actual mass-loss rate through the disc is $\dot{M}_{\mathrm{d}}$ multiplied by the solid angle of the disc divided by $4 \pi$. The velocity law is quoted in the form above with $w(r)=\left(1-R_{0} / r\right)^{\beta}$ where $R_{0}=\left(1-w_{i}\right)^{1 / \beta}$, and $w_{i}$ is the fraction of the terminal velocity that the flow has at the star. It is expected that the radial velocity at the stellar surface (corresponding to $w_{i}$ ) will be less than the sound speed. With terminal velocities $v_{\infty} \sim 100 \mathrm{~km} \mathrm{~s}^{-1}$ (Zickgraf et al. 1985) and the sound speed $\sim 10-20 \mathrm{~km} \mathrm{~s}^{-1}$ for B stars this corresponds to $w_{i} \approx 0.1$. Zickgraf (1992), estimates the disc opening angle to be 10-20 from the relative frequency of $\mathrm{sgB}[\mathrm{e}]$ stars with edge-on and polar characteristics, and this value is used hereafter.

\subsubsection{Viscous disc model}

The model of viscous outflowing discs has been discussed by Lee et al. (1991) and Okazaki (2001) and applied to classical Be star observations by Porter (1999). In this model, the angular momentum distribution within the disc is determined by viscosity, in a similar way to the more familiar accretion disc. However, in this case the angular momentum source for viscous discs is the central star (which acts as an angular-momentum sink for accretion discs). In common with the bi-stability mechanism, this model also requires that the star is a fast rotator - indeed it insists that the equatorial region is rotating at Keplerian speeds. The weak link in the model for classical Be stars (which will also feature in this application to $\mathrm{sgB}[\mathrm{e}]$ stars) is that the mechanism to inject angular momentum in to the disc at its inner regions is still largely unknown - often quoted candidates are pulsation and magnetic fields.

The solution of the equations of mass continuity and angular momentum conservation in the radial and azimuthal coordinates produce a disc which has surface-density, radial and azimuthal velocity are well represented in the inner $\sim 10^{2} R_{*}$ by power laws in radius (Okazaki 2001).

In particular the discs are close to Keplerian $v_{\phi} \propto r^{-1 / 2}$, the radial velocity increases linearly with radius $v_{\mathrm{r}} \propto r$ (and is subsonic until large radii), and the surface-density $\Sigma$ (the density integrated through the disc) decreases as $\Sigma \propto r^{-2}$. The disc flares - the scale height $H$ of the disc increases with radius as $H=c_{\mathrm{S}} r^{1.5} / \sqrt{G M_{*}}$ where $G$ is the gravitational constant, $M_{*}$ is the stellar mass and $c_{\mathrm{s}}$ is the sound speed. The viscous disc density and velocities are

$\rho_{\mathrm{d}}=\rho_{0, \mathrm{~d}}\left(\frac{r}{R_{*}}\right)^{-3.5} \exp \left(-\frac{z^{2}}{2 H^{2}}\right)$ 
$v_{\mathrm{r}, \mathrm{d}}=v_{\mathrm{r} 0, \mathrm{~d}}\left(\frac{r}{R_{*}}\right)$

$v_{\phi, \mathrm{d}}=v_{\phi 0}\left(\frac{r}{R_{*}}\right)^{-0.5}$

where $\rho_{0, \mathrm{~d}}, v_{\mathrm{r} 0, \mathrm{~d}}$ and $v_{\phi 0}$ are the density, radial velocity, and azimuthal velocity at the star-disc boundary $\left(r=R_{*}\right)$ respectively, and $z$ is the height above the equatorial plane. Also, $v_{\mathrm{r} 0, \mathrm{~d}} \lesssim 10^{-2} c_{\mathrm{s}}$ and $v_{\phi 0} \approx \sqrt{G M_{*} / R_{*}}$ In the outer parts of the disc, it becomes transonic, and angular momentum conserving (changes to $v_{\phi, \mathrm{d}} \sim r^{-1}$ ).

\subsection{Temperature profile}

The form of temperature profile for a disc is difficult to determine without a full calculation. However a simple argument can be applied to both equatorial winds and viscous discs which can provide an estimate of the radial variation of temperature. It is assumed that the disc will have a constant temperature with radius until a cooling radius $r_{c}$, and beyond this the disc will cool. In the limit that no radiation of any wavelength impinges on the disc, it will cool adiabatically such that $T \propto r^{-4 / 3}$. However, it is most likely that the disc will act as a reprocessing disc: it will absorb radiation from the star, and re-emit at its local equilibrium temperature $T_{\mathrm{d}}$. Hence the total energy emitted by the disc $\left(\propto T_{\mathrm{d}}^{4}\right)$ is proportional to the flux recieved, which in turn is proportional to $r^{-3}\left(r^{-2}\right.$ from the dilution of the radiation field, and an extra factor of $r^{-1}$ from the cosine term produced by the geometric reduction of the projected disc area). Hence these reprocessing disc have $T_{\mathrm{d}}^{4} \propto r^{-3}$ or $T_{\mathrm{d}} \propto r^{-3 / 4}$. This argument is valid for both equatorial winds, and viscous discs.

The cooling will change the metallic species responsible for the absorption and scattering of the equatorial wind and hence may lead to a more complicated discussion of the wind driving. This has already been implicitly taken into account by using the slowly accelerating beta-velocity law fitted to the UV lines from Zickgraf et al. (1996).

Cooling will effect the viscous disc: once the temperature in the viscous disc varies, then the density and velocity profiles are also affected. This is due to the torques changing as the viscosity $v=\alpha c_{\mathrm{s}} H$ alters ( $\alpha$ is the Shakura \& Sunyaev 1973 viscosity parameter). This is a result of the sound speed $c_{\mathrm{S}}$ and also the disc scale height $H \propto c_{\mathrm{s}}$ changing. To ensure mass and angular momentum conservation, the density, radial velocity and scale height change to

$\rho \propto r^{-19 / 8}$

$v_{\mathrm{r}} \propto r^{1 / 4}$

$H \propto r^{9 / 8}$

i.e. their radial dependence becomes less severe, whilst the azimuthal velocity remains Keplerian (Okazaki 2001, private communication). Therefore at radii where the viscous disc is cooling $\left(r>r_{\mathrm{c}}\right)$ Eqs. (7)-(9) replace Eqs. (4)-(6).

\section{Dust formation}

Any model which attempts to explain the properties of $\operatorname{sgB}[\mathrm{e}]$ star circumstellar matter must also provide a site for dust formation. To produce dust, two criteria need to be fulfilled: the temperature of the gas must be lower than the sublimation temperature of the dust (around $1500 \mathrm{~K}$ depending on the chemical composition of the dust), and secondly, the number density of the species involved in the formation of the dust needs to be above a critical value.

It is expected that $\mathrm{sgB}[\mathrm{e}]$ stars' dust will be silicate based, as carbon is depleted due to the CNO processing cycle in massive stars, and indeed $\mathrm{sgB}[\mathrm{e}]$ stars do show features due to silicates in their spectra (e.g. Voors 1999). Examination of the 8-13 $\mu \mathrm{m}$ spectra of R126 presented in Roche et al. (1993) shows that there are no strong emission features, which suggests silicate dust is not present. However, suppression of the silicate feature may be achieved by having large grains $\left(a_{\mathrm{d}}>\right.$ $10 \mu \mathrm{m}$ ), or by not having a stratified disc structure (leading to any optically thin "atmosphere" region) i.e. the emitting region is completely optically thick (see Dullemond et al. 2001 and Meeus et al. 2002).

Gail \& Sedlmyar (1988) showed that a critical number density can be derived by comparing the timescale for the chemical reactions responsible for dust formation $\tau_{\mathrm{ch}}$ to the timescale for a parcel of gas to change in density and temperature $\tau_{\exp }$ (i.e. an expansion timescale). Grain growth will occur if $\tau_{\mathrm{ch}}<\tau_{\text {exp }}$. Gail \& Sedlmyar find that this occurs for any size of grain if the number density of the growth species is larger than a minimum density

$n_{\min }=\frac{v_{\mathrm{r}}}{10^{-16} r v_{\text {rel }}}$

where $v_{\text {rel }}$ is the relative velocity between the growing grain cluster and a molecule (set to the thermal velocity here). It is assumed that dust formation occurs if $\epsilon \rho(r) /\left(\mu m_{\mathrm{p}}\right)>n_{\min }$ (where the ratio of relevant species to total number density is $\epsilon$ and $\mu \approx 1.25^{-1}$ is the mean molecular weight for neutral gas). This criterion may be compared to differing models of the disc component around $\mathrm{sgB}[\mathrm{e}]$ stars.

The value of $\epsilon$ is taken to be $\epsilon=10^{-5}$ corresponding to $1 / 3$ of Solar silicon abundance appropriate for the LMC metallicity. As an example calculation of $n_{\text {nim }}$, the sound speed (approximating to $\left.v_{\text {rel }}\right)$ has been calculated assuming that for both the equatorial wind and the viscous disc models, cooling takes place beyond $r_{\mathrm{c}}=1.5 R_{*}$, and that the temperature profile is $T \propto\left(r / r_{\mathrm{c}}\right)^{-3 / 4}$ thereafter. Finally the temperatures at $r=R_{*}$ are taken to be $0.8 T_{\text {eff }}$ and $0.5 T_{\text {eff }}$ for the equatorial wind and disc models respectively. The results of this calculation are shown in Fig. 1.

\subsection{Equatorial wind model}

The equatorial wind is assumed to have the same parameters as described in Sect. 2.1.1: $\left(\beta=4\right.$ and $\left.w_{i}=0.1\right)$ and a massloss rate of $\dot{M}_{\mathrm{d}}=10^{-5} M_{\odot} \mathrm{yr}^{-1}$ (this rate has been chosen as it produces a dense enough disc to acccount for the near-IR emission of the model star R126 (see Sect. 5). Figure 1 shows the number density of this wind model as well as the critical number density required to form dust. It can be seen that dust can only form for $r<50 R_{*}$. The arrow in Fig. 1 shows the radius where the temperature falls to $1500 \mathrm{~K}$ - this occurs at 


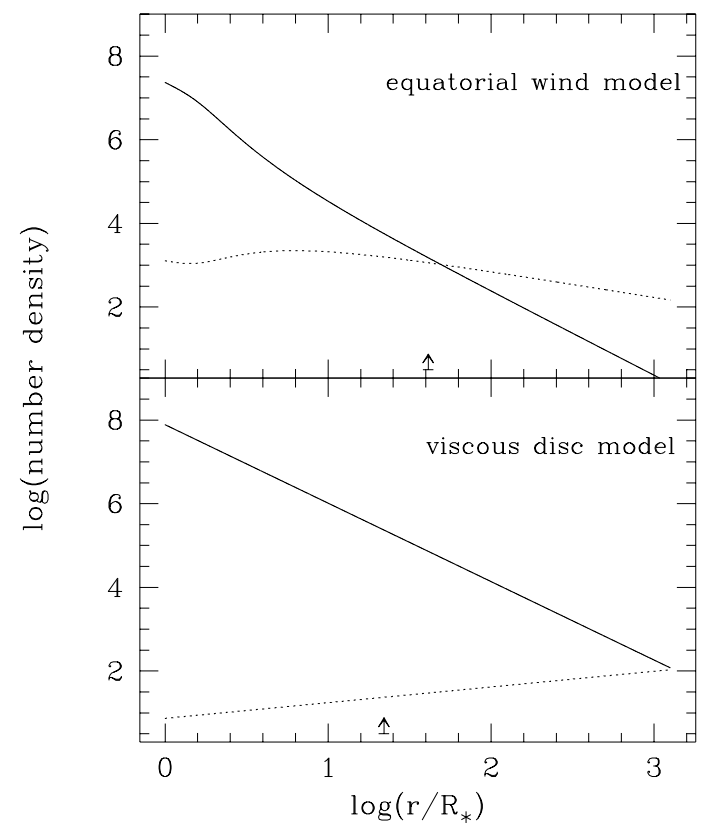

Fig. 1. Comparison of number density of carbon (solid line) with $n_{\min }$ from Eq. (10) (dotted line): top panel, the equatorial wind model described in the text, lower panel, the viscous disc model. Dust may form in the region where the number density is larger than $n_{\min }$ only if the flow is cool enough. The arrows indicate the radius at which the temperature has fallen to $1500 \mathrm{~K}$.

a radius of $41 R_{*}$. Therefore there exists a region from $\sim 40-$ $50 R_{*}$ where the gas is dense enough and cool enough for dust formation.

\subsection{Viscous disc model}

Figure 1 also shows the number density within a viscous disc with $\rho_{0}=10^{-11} \mathrm{~g} \mathrm{~cm}^{-3}$ (again chosen to reproduce the near-IR emission, see Sect. 5) and $v_{\mathrm{r} 0}=0.3 \alpha T_{4} \sqrt{R_{*} / M_{*}} \mathrm{~km} \mathrm{~s}^{-1}$ (where $\alpha=0.1$ is the viscosity parameter, $T_{4}$ is the disc temperature in $10^{4} \mathrm{~K}$ and $R_{*}$ and $M_{*}$ are in Solar units - see Eq. (6) of Porter 1998). Again the cooling radius is set to $r_{\mathrm{c}}=1.5 R_{*}$. For $r \lesssim 1250 R_{*}$ the number density in the disc is high enough to enable dust to form. The radius where the temperature falls to $1500 \mathrm{~K}$ is $23 R_{*}$, well within the region where dust may form.

\subsection{Dust formation conclusions}

Both the viscous disc model and the wind model have a region where their number density is larger than the critical value for dust formation, implying that both models can form dust, with the proviso that the temperature must also be below the sublimation temperature $T_{\text {dust }}$. The major difference between the models is for $n_{\min }$, which is significantly lower for the viscous disc model as its radial velocity is far lower than for the equatorial wind model. Both models are successful in providing a site for dust formation, and so both are still good candidates for the actual discs of $\mathrm{sgB}[\mathrm{e}]$ stars.

\section{Continuum emission}

The continuum emission is now calculated from the density fields in the models and compared to observations of R126. The model includes a fast polar wind (with a fixed mass-loss rate of $10^{-5} M_{\odot} \mathrm{yr}^{-1}$ ), the disc models described in the last section, and dust (where it exists) in the disc. Although the fast polar wind is included, its contribution to the final flux is negligible, and is only included for completeness. In order to calculate the excess emission in the optical - IR, the prescription of Waters (1986) is used. The star is assumed to be pole-on.

The viscous disc model requires that the density $\rho_{0, \mathrm{~d}}$, the dust formation temperature $T_{\text {dust }}$ and the cooling radius $r_{\mathrm{c}}$ are specified to calculate the emission. The equatorial wind model requires a mass-loss rate $\dot{M}_{\mathrm{d}}$, a terminal velocity $v_{\infty, \mathrm{d}}$, the value of $\beta$ for the velocity law and a flow opening angle, as well as the dust temperature and cooling radius: $\beta$ is fixed at $\beta=4$, the terminal velocity $v_{\infty}=70 \mathrm{~km} \mathrm{~s}^{-1}$, and the opening angle to $20^{\circ}$ hereafter.

The number density is calculated where the temperature falls below $T_{\text {dust }}$ and compared to the minimum number density for dust formation from Eq. (10). If dust is found to form, the optical depth through the disc at that radii is

$\tau_{\text {dust }}=\int_{\text {disc }} \rho \kappa_{\text {dust }} \mathrm{d} z$

where $\rho$ is the gas density, and $\kappa_{\text {dust }}$ is the opacity of the dust. The opacity used is an approximate fit to that calculated for a distribution of grain sizes (e.g. see Wood et al. 2002). A fit to Wood et al.'s data for Solar metallicity in their Fig. 2 from $0.3-100 \mu \mathrm{m}$ produces $\kappa_{\text {dust }}=120(\lambda / 0.1 \mu \mathrm{m})^{-0.6} \mathrm{~cm}^{2} \mathrm{~g}^{-1}$. The opacity used here is $1 / 3$ of this, to take into account the lower metallicity of the LMC. Therefore the dust opacity used here is

$\kappa_{\text {dust }}=40\left(\frac{\lambda}{0.1 \mu \mathrm{m}}\right)^{-0.6} \mathrm{~cm}^{2} \mathrm{~g}^{-1}$.

This simple expression for dust opacity is used so that the reason for the failure of the standard viscous disc and equatorial wind models - see below - is clear. In order that the dust is readily observed in the spectrum, it is expected that the optical depth of the dust will be $\tau_{\text {dust }}>1$.

The emitted flux is then integrated over the disc area, which, for face-on discs, produces

$F_{v}=\frac{1}{D^{2}} \int_{R_{*}}^{R_{\mathrm{disc}}} \pi B_{v}[T(r)]\left(1-\mathrm{e}^{-\tau_{\text {dust }}}\right) 2 \pi r \mathrm{~d} r$

where $R_{\text {disc }}$ is the outer edge of the disc, and $D$ is the distance to the star and $B_{v}[T(r)]$ is the Planck function.

\section{Results}

The UBVRIJHKLMN photometry for R126 is taken from Zickgraf et al.'s (1985) compilation, and is supplemented by $12 \mu \mathrm{m}$ and $25 \mu \mathrm{m}$ IRAS points from Schwering (1989). The reddening to the object was taken to be $E(B-V)=0.25$ from Zickgraf et al. (1985). The calculated emission from the model has been reddened with the expression given in Howarth (1983) 


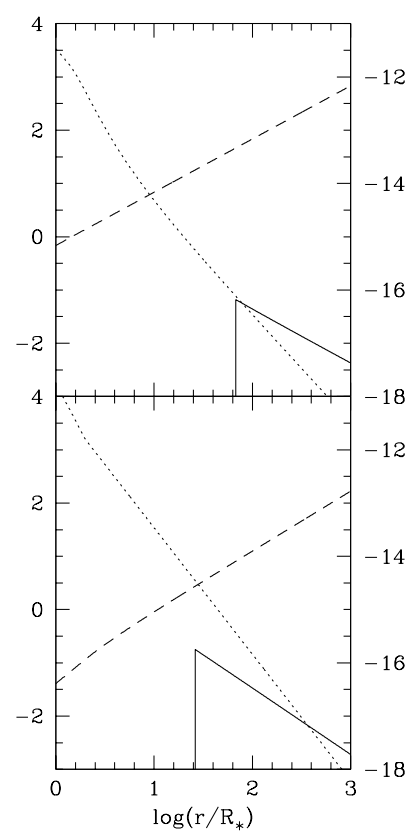

Fig. 2. Dust optical depths within the discs at a wavelength of $5 \mu \mathrm{m}$ for the two "standard" models. The solid line is the log of the optical depth (referring to the left hand scale), the dashed line is the log of the disc scale height $H$ in stellar radii (again referring to the left hand scale), and the dotted line is the log of the disc density in $\mathrm{g} \mathrm{cm}^{-3}$ (the right hand scale). The top panel is for the equatorial wind model, the bottom panel for the viscous disc model.

and the model flux in the $V$ band has been normalised to the $\mathrm{R} 126$ observations for all models. The underlying photospheric continuum is a Kurucz $22000 \mathrm{~K}, \log g=3.0$ ATLAS9 model.

The proceedure used to fit the photometry involves a search over the mass-loss rate $\dot{M}_{\mathrm{d}}$, dust formation temperature and cooling radius for the equatorial wind model. The viscous disc model has the density normalization $\rho_{0}$, dust formation temperature and cooling radius varied to find the best fit. The results are summarised in Table 1: the various parameters for the models are given, along with their $\chi^{2}$ evaluation of fit. Best-fit values to the optical-near-IR data produces values of $\dot{M}_{\mathrm{d}}=1.1 \times 10^{-5} M_{\odot} \mathrm{yr}^{-1}$ and $\rho_{0}=10^{-10.75} \mathrm{~g} \mathrm{~cm}^{-3}$ for both models respectively. The equivalent mass loss rate for the viscous disc model is $7 \times 10^{-7} M_{\odot} \mathrm{yr}^{-1}$ : although it appears counterintuitive that this is less than the polar (fast wind) mass loss rate, it is reconciled in that the mechanism for mass ejection into the viscous disc is not the same as over the poles (indeed the cause for mass injection in to the disc it is still unknown). Hence the equatorial mass loss rate need not bear any relation to the polar mass loss rate.

There is a significant problem with the dust emission for both models in that the optical depths where dust forms is not large enough to generate the observed emission. To illustrate this, Fig. 2 shows the dust optical depth $\tau_{\text {dust }}$ (and density and disc scale-height) for both models as a function of radius. It can be clearly seen that the optical depth is significantly less than unity at the dust formation radius, and decreases with radius. Therefore it might be expected that the continuum emission will not be able to reproduce the obserevational data. Figure 3

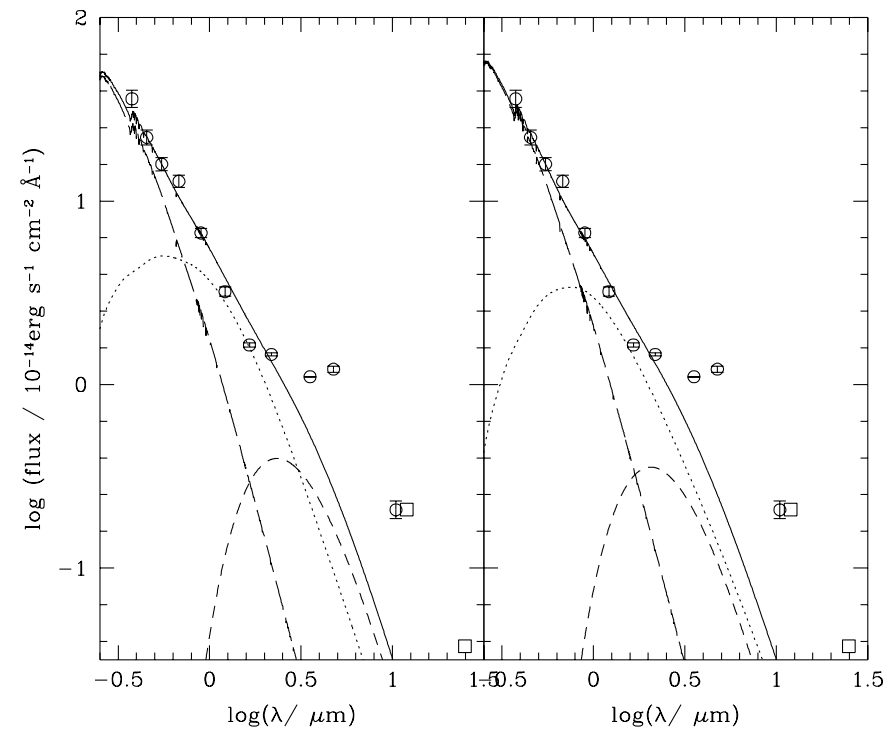

Fig. 3. Continuum emission from the star (R126) and the two best-fit models. The total model flux (solid line) consists of Kurucz model atmosphere (long dashed line), free-free and free-bound emission (dotted line) and dust emission (short-dashed line) for the "standard" models. The model has been normalised to the R126 data of Zickgraf et al. (1985) in the $V$ band. The left panel is for the equatorial wind model, and the right panel is for the viscous disc model. Model parameters are given in Table 1.

displays the full spectrum for both models. As can be seen the optical to near-IR flux is reproduced, although longward of $\sim 2 \mu \mathrm{m}$ the dust emission is too low by about an order of magnitude.

How may this be remedied? Or are the two proposed models to be rejected? To produce the observed emission, the optical depth $\tau_{\text {dust }}$ needs to be increased by one to two orders of magnitude. For these models this requires a significant increase in density. A global increase in the density (corresponding to increases in $\dot{M}_{\mathrm{d}}$ and $\rho_{0}$ ) would produce too much emission in the optical-near-IR range, and so may be ruled out. Increasing the opening angle for the equatorial wind model (or decreasing the terminal velocity) will also produce the same effect of increasing the emission at all wavelengths, and not simply the dust emission. To investigate how dependent the models are to the input assumptions, several changes are made in turn to the standard models: the temperature power law indices are made free parameters, the dust opacity is set to that appropriate to Solar metallicity, and finally, the density power law indices are made free parameters.

\subsection{The temperature profile}

It is possible that the temperature profile that has been used is not correct, although the disc must cool in order for it to form dust. To assess whether the observations may be fitted using a different temperature profile, beyond the cooling radius, the power law $T_{\mathrm{d}} \propto r^{-m}$ has been varied using different values of $m$, from 0.4 to 1.5 and the best fit models calculated. The best-fit parameters are shown in Table 1. The temperature-power law 
Table 1. The parameters for the best-fit models. The first two models are the standard models with $m$ fixed at 0.75 . The following two models have the temperature power law index $m$ as a free parameter in the search and with the inner flow parameters as above. Models $5 \& 6$ have an enhanced dust opacity appropriate for Solar metallicity. The final two models have a power law in density as stated - see text.

\begin{tabular}{lrrrrlr}
\hline \hline Model & $m$ & $r_{\mathrm{c}} / R_{*}$ & $T_{\text {dust }}(\mathrm{K})$ & $\kappa_{\text {dust }}(0.1 \mu \mathrm{m})$ & density & $\chi^{2}$ \\
\hline 1. Wind & 0.75 & 2.3 & 1400 & 40 & $\dot{M}_{\mathrm{w}}=1.1 \times 10^{-5} M_{\odot} \mathrm{yr}^{-1}$ & 407 \\
2. Viscous disc & 0.75 & 2.0 & 1600 & 40 & $\rho=10^{-10.75}\left(r / R_{*}\right)^{-3.5} \mathrm{~g} \mathrm{~cm}^{-3}$ & 403 \\
3. Wind & 0.60 & 1.1 & 1400 & 40 & $\dot{M}_{\mathrm{w}}=1.1 \times 10^{-5} M_{\odot} \mathrm{yr}^{-1}$ & 359 \\
4. Viscous disc & 0.50 & 2.1 & 1500 & 40 & $\rho=10^{-10.7}\left(r / R_{*}\right)^{-3.5} \mathrm{~g} \mathrm{~cm}^{-3}$ & 397 \\
5. Wind & 0.75 & 2.0 & 1300 & 120 & $\dot{M}_{\mathrm{w}}=1.1 \times 10^{-5} M_{\odot} \mathrm{yr}^{-1}$ & 316 \\
6. Viscous disc & 0.75 & 2.9 & 1400 & 120 & $\rho=10^{-10.7}\left(r / R_{*}\right)^{-3.5} \mathrm{~g} \mathrm{~cm}^{-3}$ & 313 \\
7. Wind & 0.75 & 3.5 & 700 & 40 & $\rho=10^{-11.9}\left(r / R_{*}\right)^{-1.7} \mathrm{~g} \mathrm{~cm}^{-3}$ & 64 \\
8. Viscous disc & 0.75 & 6.5 & 800 & 40 & $\rho=10^{-11.0}\left(r / R_{*}\right)^{-2.7} \mathrm{~g} \mathrm{~cm}^{-3}$ & 61 \\
\hline
\end{tabular}

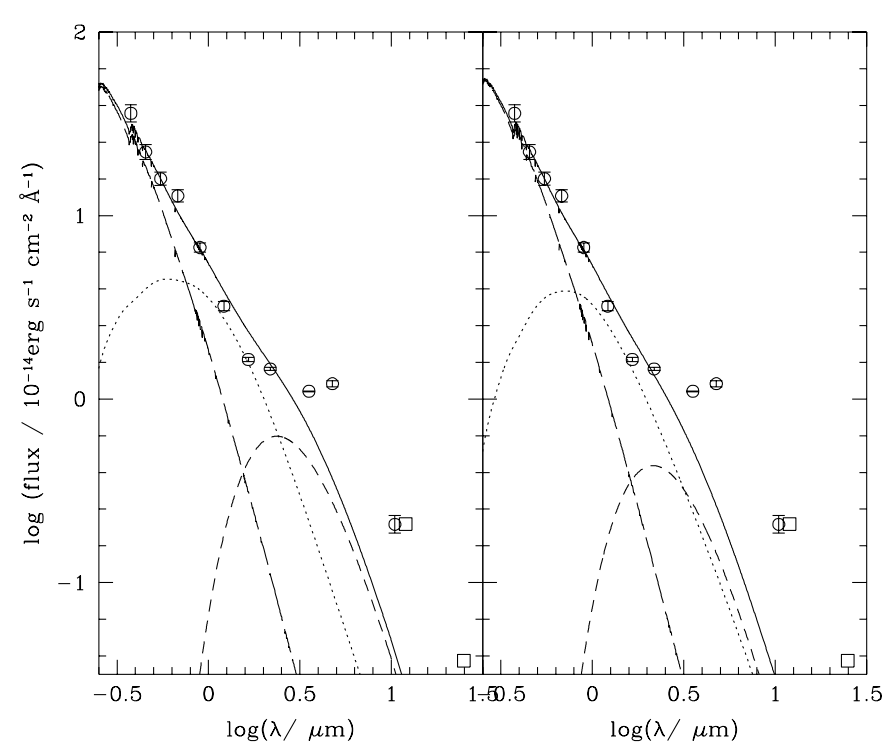

Fig. 4. As Fig. 3 except that the temperature power law index is a free parameter. The left panel is for the equatorial wind model with $T \propto r^{-0.6}$, and the right panel is for the viscous disc model $T \propto r^{-0.5}$. Further model parameters are given in Table 1. Comparison with Fig. 3 illustraltes that the effect of allowing the temperature power law to change is negligible.

index of the best models is $m=0.6$ for the equatorial wind and $m=0.5$ for the viscous disc.

In the case of the equatorial wind the best-fit dust temperature is identical to the standard wind model (1400 K), and the cooling radius is slightly smaller. However, the fit is again characterised by a significant underestimate of the dust emission indicating that variation of the temperature power law produces a negligible effect on the resultant spectrum. The viscous disc produces a slightly cooler dust formation temperature $(1500 \mathrm{~K})$ than in the standard case $(1600 \mathrm{~K})$. However, as in the wind case, the fit is barely improved with significant underestimate of the dust emission.

Both of these models are presented in Fig. 4. It appears that the failure of the standard models to reproduce the observations is not dominated by the temperature profile in either model.

\subsection{The dust opacity}

Whilst so far the opacity from Wood et al. (2002) has been used, corrected to take account of the lower metallicity of the LMC, here the normalization for Solar metallicity is used (i.e. the LMC star R126 is assumed to have Solar metallicity). This illustrates the effect of increasing the opacity, whilst keeping the form of the density field the same as in the standard cases.

The dust opacity used here is $\kappa_{\text {dust }}=120$ $(\lambda / 0.1 \mu \mathrm{m})^{-0.6} \mathrm{~cm}^{2} \mathrm{~g}^{-1}$, and the best-fitting models are shown in Fig. 5 (with parameters in Table 1: models 5 and 6). Formally, from the $\chi^{2}$ values in Table 1, it can be seen that increasing the dust opacity decreases the residuals. However, the best fitting models (Fig. 5) are still woefully inadequate in accounting for the dust emission.

Further increase in the dust opacity should provide a high enough optical depth to account for observations. However, this would require an exceptionally large metallicity for this star which is physically very difficult to justify at this stage of evolution of the star, and therefore this possibility is disregarded in favour of the dynamical arguments given later.

\subsection{The density profile}

The standard density profiles for the wind model (Eq. (1)) is now changed to a pure power law form: $\rho / \rho_{0}=\left(r / R_{*}\right)^{-\mathrm{n}}$ (where $\rho_{0}$ is a normalization density at $r=R_{*}$ ). The sole difference between the two models now is (i) that the equatorial wind model has a disc with a constant opening angle $\left(20^{\circ}\right)$, whereas the viscous disc flares, and (ii) that the density falls exponentially with height away from the equatorial plane for the viscous disc. Only the temperature power law index is fixed (with $m=0.75$ ), and the best-fit model search is conducted over dust temperature $T_{\text {dust }}$, density power law index $n$, density normalization $\rho_{0}$, and cooling radius $r_{\mathrm{c}}$.

The best-fitting equatorial wind model is shown in Fig. 6, and provides an excellent description of the continuum flux of R126. The parameters of this fit are $n=1.7$ and $\rho_{0}=$ $10^{-11.9} \mathrm{~g} \mathrm{~cm}^{-3}$, cooling radius of $r_{\mathrm{c}}=3.5 R_{*}$ and dust temperature of $T_{\text {dust }}=700 \mathrm{~K}$. A literal interpretation of this best 


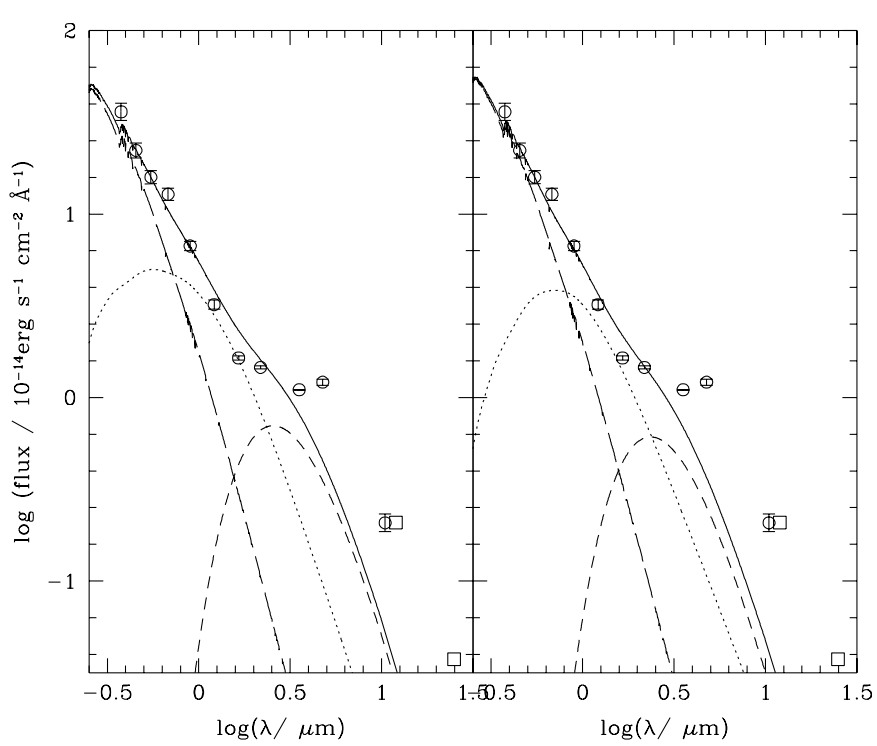

Fig. 5. As Fig. 3 except that the dust opacity normalization is appropriate for Solar metallicity: $\kappa_{\text {dust }}=120(\lambda / 0.1 \mu \mathrm{m})^{-0.6} \mathrm{~g} \mathrm{~cm}^{-3}$. Further model parameters are given in Table 1 . The left panel is for the equatorial wind model and the right panel is for the viscous disc model.

fit solution would predict that the radial velocity actually decreases with radius (from comparison with Eq. (1)), which indicates that the simple wind model is an erroneous description of the actual disc around R126.

The best fiting viscous disc model is also shown in Fig. 6 and also provides a good fit to the data. The density of this model is $\rho_{0}=10^{-11.0} \mathrm{~g} \mathrm{~cm}^{-3}$ (note this is significantly higher than the best fitting equatorial wind model), and the density power law index is $n=2.7$, the cooling radius is $r_{\mathrm{c}}=6.5$ and the dust formation temperature is $T_{\text {dust }}=800 \mathrm{~K}$.

For both the equatorial wind, and the viscous disc the $\chi^{2}\left(n, \rho_{0}, r_{\mathrm{c}}, T_{\text {dust }}\right)$ values have been inspected for different models around this minimum, in order to estimated the variation in the parameters. A value of $\chi^{2}$ is obtained which is twice the best fitting model if parameter uncertainties are $\delta n \sim \pm 0.1$, $\delta \rho_{0} \sim \pm 0.1 \delta T_{\text {dust }} \sim \pm 100 \mathrm{~K}$, and $\delta r_{\mathrm{c}} \sim \pm 3$. Clearly the least constrained parameter is the cooling radius.

\section{Discussion}

It appears from the results that both the "standard" equatorial wind model and the viscous disc model have trouble in generating the continuum emission for $\operatorname{sgB}[\mathrm{e}]$ stars. To do so, the density field has had to be changed somewhat from initial theoretical expectations. The deviations from the standard density structures of the models may indeed highlight some aspects of the two models which have not been considered here. The equatorial wind may be clumped at large radii, hence producing parts of the disc which have a large dust optical depth. However, the total emission may not necessarily increase due to the subsequent reduction in effective emitting area (i.e. if the gas is clumped into dense regions, the "filling factor" of that dense gas degreases). The low power law index for the best fitting viscous disc model ( $n=2.7$ compared to $n=3.5$ for the

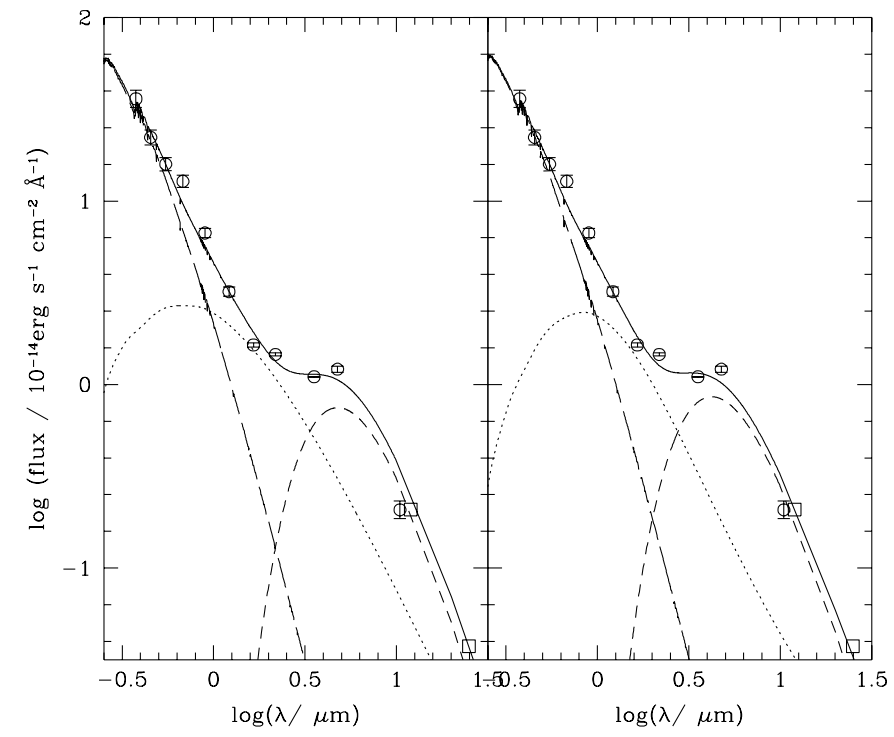

Fig. 6. As Fig. 3 except that the density power law is a free parameter. The left panel is for the equatorial wind model with $\rho=$ $10^{-11.9}\left(r / R_{*}\right)^{-1.7} \mathrm{~g} \mathrm{~cm}^{-3}$, and the right panel is for the viscous disc model $\rho=10^{-11.0}\left(r / R_{*}\right)^{-2.7} \mathrm{~g} \mathrm{~cm}^{-3}$. Other model parameters are given in Table 1.

standard model) might indicate an extra dependence of the viscosity (and perhaps the $\alpha$ parameterization) on the surface density, or the disc temperature which has not been investigated here.

Assuming that either of these models can account for all the continuum emission, can the two models both produce the observed heavy element line emission from $\mathrm{sgB}[\mathrm{e}]$ stars? The equatorial wind model was introduced to do exactly that (e.g. see Zickgraf et al. 1996), and the line emission comes from the equatorial wind flow itself. Therefore, this model would seem to provide a complete description of the disc around a $\mathrm{sgB}[\mathrm{e}]$ star. However, there is a difficult issue for the equatorial wind model: the electron scattering optical depth becomes larger than unity at relatively small radii. Whilst high continuum optical depths are commonplace for (approximately) spherically symmetric Wolf-Rayet winds, in this asymmetric case it may lead to problems in driving the wind. Photons cannot physically penetrate into the disc which leads to little or no radiative driving for the gas, and hence the density profile may not actually allow the gas to be driven! This might cast doubt on whether the equatorial wind model is correct in principle. However, this may also highlight that the wind may not have a velocity law typified by the "beta" parameterization.

The production of the heavy element line emission from the viscous disc model is slightly more complicated. The dominant velocity component in the disc is a rotational one, and therefore optically thin emission lines should exhibit double-peaked profiles (similar to those observed in classical Be stars). However, the model in Oudmaijer et al. (1998), provides a separate site for line emission with a dominant radial component of velocity: a disc wind. Here, the emission from the reprocessing disc helps to drive a flow from the upper and lower surfaces of the disc to relatively modest terminal velocities $\sim 100 \mathrm{~km} \mathrm{~s}^{-1}$. 
The resultant line emission may have a double-peaked disc component, but will have a large radial component imposed on it too. Currently, no attempt has been made to synthesize line profiles from the work presented by Oudmaijer et al. An issue to resolve here is whether there is enough flow generated in the disc-wind to account for the lines observed in $\mathrm{sgB}[\mathrm{e}]$ stars.

A weakness for the viscous disc model is that the source of angular momentum required to supply the disc is still unidentified (although candidates exist: pulsation and/or magnetic fields). Until a coherent theory of angular momentum transfer from the star to the disc is produced, then the viscous disc model will only remain a promising candidate. Note that if the viscous disc model is the correct structure, then it is possible that the way in which classical Be stars supply angular momentum to their discs is the same for $\operatorname{sgB}[\mathrm{e}]$ stars. In this case then the $\mathrm{sgB}[\mathrm{e}]$ stars will be invaluable as they have a different envelope structure than classical Be stars, but it might be likely that both supply angular momentum in the same fashion.

From the results above, it is difficult to make a strong case which will prefer one model over the other, or even that either model is appropriate. Clearly, the discs of $\operatorname{sgB}[\mathrm{e}]$ stars are not the simple radiation driven flows, nor the simple viscous discs that have been previously suggested.

\section{Conclusion}

Both of the models that have been presented in this study are apparently incapable of reproducing the observational results in their simplest form. Whilst both meet the requirements for the formation of dust they are unable to yield the necessary broad-band fluxes.

The first model is the enhanced equatorial flow from Lamers \& Pauldrach's bi-stability theory (1991); the other is the viscous disc model. The densities are alike, but the velocity fields are disparate. Both competing paradigms expect that the central supergiant star is rotating at a significant fraction of its break-up speed.

By relaxing several assumptions (regarding the temperature profile, the dust opacity, and the density profile), the applicability of the models have been tested. It is found that the models are unable to reproduce the observations unless the disc density field is significantly altered, although then a good representation of the observations can be obtained.

There are possibilities for both models to produce the observed permitted and forbidden line emission: the equatorial wind model produces the emission itself, and the viscous disc model produces a disc-wind from it's surfaces which may produce the lines (although this remains untested).

In both the equatorial wind model and the viscous disc model, there is still work to be undertaken to understand the form of the density profile necessary to account for observations. Clearly both models in their simplest form are inadequate as a description of the discs of $\operatorname{sgB}[\mathrm{e}]$ stars.

Acknowledgements. JMP would like to thank Simon Clark, Atsuo Okazaki, Toby Moore and Lee Howells for help in preparing and commenting on previous drafts of this paper, and Jon Bjorkman for his useful input regarding dust emission. JMP also thanks the anonymous referees for making useful comments on the submitted versions.

\section{References}

Bjorkman, J. E. 1998, in B[e] stars, ed. A. M. Hubert, \& C. Jaschek (Kluwer Academic Publishers), 194

Bjorkman, J. E., \& Cassinelli, J. P. 1993, ApJ, 409, 429

Castor, J. I., Abbott, D. C., \& Klein, R. I. 1975, ApJ, 195, 157

Dullemond, C. P., Dominik, C., \& Natta, A. 2001, ApJ, 560, 957

Gail, H. P., \& Sedlmayr, E. 1988, A\&A, 206, 153

Howarth, I. D. 1983, MNRAS, 203, 301

Kudritzki, R. P., Pauldrach, A., Puls, J., \& Abbott, D. C. 1989, A\&A, 219, 205

Lamers, H. J. G. L. M., \& Pauldrach, A. W. A. 1991, A\&A, 244, L5

Lamers, H. J. G. L. M., Zickgraf, F.-J., de Winter, D., Houziaux, L., \& Zorec, J. 1998, A\&A, 340, 117

Lee, U., Saio, H., \& Osaki, Y. 1991, MNRAS, 250, 432

Meeus, G., Bouwman, J., Dominik, C., Waters, L. B. F. M., \& de Koter, A. 2002, A\&A, 392, 1039

Okazaki, A. T. 2001, Publ. Ast. Soc. Japan, 53, 119

Oudmaijer, R. D., Proga, D., Drew, J. E., \& de Winter, D. 1998, MNRAS, 300, 170

Owocki, S. P., Cranmer, S. R., \& Gayley, K. G. 1996, ApJ, 472, L115

Pelupessy, I., Lamers, H. J. G. L. M., \& Vink, J. S. 2000, A\&A, 359, 695

Petrenz, P., \& Puls, J. 2000, A\&A, 358, 956

Porter, J. M. 1998, A\&A, 333, L83

Porter, J. M. 1999, A\&A, 341, 560

Roche, P. F., Aitken, D. K., \& Smith, C. H. 1993, MNRAS, 262, 301

Shakura, N. I., \& Sunyaev, R. A. 1973, A\&A, 24, 337

Schwering, P. B. W. 1989, A\&AS, 79, 105

Vink, J. S., de Koter, A., \& Lamers, H. J. G. L. M. 1999, A\&A, 350, 181

Voors, R. H. M. 1999, Ph.D. Thesis, Utrecht University

Waters, L. B. F. M. 1986, A\&A, 162, 121

Wood, K., Wolff, M. J., Bjorkman, J. E., \& Whitney, B. 2002, ApJ, 564,887

Zickgraf, F.-J. 1992, in Nonisotropic and Variable Outflows from Stars, ed. L. Drissen, C. Leitherer, \& A. Nota, ASP Conf. Ser., 22,75

Zickgraf, F.-J., Humphreys, R. M., Lamers, H. J. G. L. M., et al. 1996, A\&A, 315, 510

Zickgraf, F.-J., \& Schulte-Ladbeck, R. E. 1989, A\&A, 214, 274

Zickgraf, F.-J., Wolf, B., Stahl, O., Leitherer, C., \& Klare, C. 1985, A\&A, 143, 421

Zickgraf, F.-J., Wolf, B., Stahl, O., Leitherer, C., \& Appenzeller, I. 1986, A\&A, 163, 119 